\title{
Treatment selection for coronary artery disease: The collision of a belief system with evidence
}

\author{
Peter K. Smith, MD
}

Development of interventional therapy for coronary artery disease (CAD) has unquestionably improved and prolonged the lives of millions, but the complex manifestations and presentations of the disease have confounded easy decision making to develop "the best treatment" option for each patient. Two important papers, recently published, support coronary artery bypass grafting $(\mathrm{CABG})$ as the treatment of choice for patients with advanced CAD (3-vessel disease or left main coronary disease) compared with percutaneous coronary intervention (PCI).

The Synergy between PCI with Taxus and Cardiac Surgery (SYNTAX) trial $^{1}$ demonstrated a significant reduction in the composite rate of major adverse cardiovascular event (MACE) end point, defined as mortality, myocardial infarction, stroke, or reintervention, for $\mathrm{CABG}$ compared with PCI. The "Appropriateness Criteria for Coronary Revascularization,' sponsored by the American College of Cardiology Foundation, assembled experts to create 180 different clinical vignettes to represent a cross-section of contemporary practice as encountered by working cardiologists and surgeons. ${ }^{2,3}$ For 3-vessel disease, $C A B G$ was rated appropriate, and PCI rated uncertain. For left main coronary disease, $C A B G$ was rated appropriate, and PCI was rated inappropriate.

Despite these findings, it is apparent that their translation into practice is being heavily influenced by various stakeholders whose belief systems are unfulfilled by the evidence. The purpose of this editorial is to clarify the body of evidence as it exists today so that all stakeholders are held accountable to the primary stakeholders, our patients.

This is only reasonable, because 3-three vessel CAD afflicts $23 \%$ of patients found to have significant coronary disease at diagnostic cardiac catheterization (unpublished data from 10,149 patients in the Duke Cardiovascular Disease Databank, 2000 to 2008). Long-term follow-up of patients who received medical treatment shows survival at 5, 10, and 15 years of only $61 \%, 38 \%$, and $22 \%$, respectively (Fig 1). When patients were treated by PCI or CABG, survival at 5, 10, and 15 years significantly improved to

From the Division of Thoracic Surgery, Duke University, Durham, North Carolina. This article is being co-published simultaneously in The Journal of Thoracic and Cardiovascular Surgery and The Annals of Thoracic Surgery.

Received for publication March 5, 2009; revisions received March 9, 2009; accepted for publication March 10, 2009.

Address correspondence to Peter K. Smith, MD, Division of Thoracic Surgery, Duke University, PO Box 3442, 4532 Hosp South, Durham, NC 27710 (E-mail: smith058@mc.duke.edu).

J Thorac Cardiovasc Surg 2009; 137:1050-3

$0022-5223 / \$ 36.00$

Copyright (c) 2009 by The American Association for Thoracic Surgery and The Society of Thoracic Surgeons

doi:10.1016/j.jtcvs.2009.03.043
$76 \%, 54 \%$, and $34 \%$, respectively (Fig 1). Logically, therefore, preservation of life should be the predominant component framing the evidence in recommending PCI or $\mathrm{CABG}$ for these patients.

\section{THE SYNTAX TRIAL}

The SYNTAX trial, organized by an international group of Western Europeans and North Americans and sponsored by industry, addressed a more focused question to determine the relative merits of PCI with drug-eluting stents or CABG for patients with 3-vessel or left main CAD. ${ }^{1}$ The randomized, prospective controlled trial screened 4337 patients at 85 sites. After evaluation, 3075 patients remained eligible for the trial, and each patient was evaluated by an interventional cardiologist and a cardiac surgeon. A total of 1800 patients were deemed suitable for PCI or CABG and were randomized and received the assigned treatment. Of the remaining 1275 patients for whom only one treatment was recommended, 1077 received CABG $(85 \%)$ and 198 received PCI $(15 \%)$ and were followed up in an observational study.

The study failed to meet its primary end point, with a relative hazard of 1.44 for excess MACE with PCI. This was statistically significant and exceeded the prespecified noninferiority magnitude. There was excess hazard for stroke with CABG of $2.2 \%$ at 1 year vs $0.6 \%$ with PCI, with half the CABG strokes occurring 3 months or longer after the procedure. The relative hazard for 1-year mortality with PCI was $1.26(P=\mathrm{NS})$.

Randomized trials generally show that $\mathrm{CABG}$ is superior to PCI for durability of effectiveness (need for reintervention) and equal or superior to PCI for mortality outcome. A short-term mortality advantage may not be demonstrable because it is offset by an increased periprocedural risk of stroke and death in CABG patients. Further follow-up demonstrates that the durability of $\mathrm{CABG}$, notably the left internal mammary artery (LIMA) anastomosis to the left anterior descending coronary artery (LAD), translates into a survival advantage. This advantage particularly applies to patients who have extensive CAD and typical comorbid risk factors.

Randomized trials are extremely valuable sources of evidence, but have important limitations. The primary limitation is generalizability, because the trial results are strictly applicable to patients who meet the enrollment criteria and trial conditions. In trials of PCI compared with CABG, the surgeon and cardiologists generally must agree that either treatment is applicable, which can eliminate up to $98 \%$ of patients. ${ }^{4}$ The SYNTAX trial partially overcomes this limitation (58\% enrollment of screened patients). It should be emphasized that the process of surgeon-cardiologist agreement on amenability to either procedure tends to make 


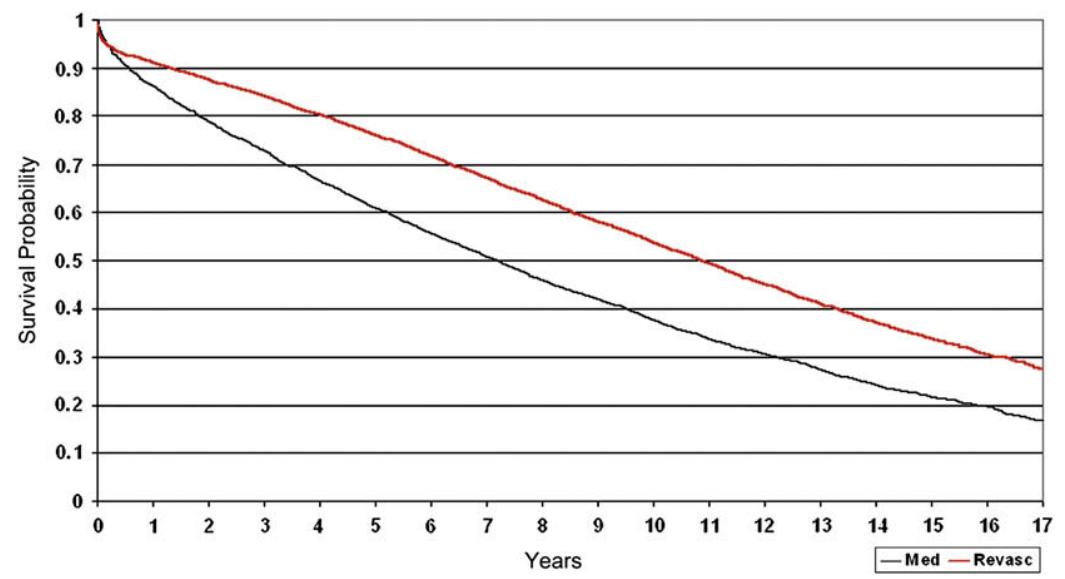

FIGURE 1. Adjusted long-term actuarial survival for 18,481 Duke University patients (1986 to 2003) with severe coronary artery disease treated with medical therapy (black line) compared with revascularization by percutaneous coronary intervention or coronary artery bypass grafting (red line).

enrolled patients more ideal for either, driving an equivalency result but also failing to reflect real-world decision making as it exists today.

A second limitation of randomized trials is inadequate statistical power to determine low-frequency safety outcomes such as death or stroke. This limitation is related to trial expense, trial objectives, trial duration, and lack of equipoise in enrollment. Power is reduced by short duration and early reporting, as exemplified by SYNTAX. Most CABG adverse events occur early, whereas many PCI adverse events occur later. Thus, conclusions based on 1-year follow-up results are heavily weighted in favor of PCI and are reported at a time when conclusions are unwarranted for a disease that claims the lives of $66 \%$ of the patients within 15 years (unpublished data from the Duke Cardiovascular Disease Databank).

A third limitation relates to trial design, which may not reflect real-world clinical treatment and may not effectively isolate the treatment effect to the primary outcome of the procedure being studied. In SYNTAX, for example, a large difference in secondary prevention medical therapy resulted from the trial design. CABG patients were significantly undertreated with aspirin (84\% vs $91 \%$ ), thienopyridine $(15 \%$ vs $71 \%)$, and statin drugs ( $75 \%$ vs $87 \%)$ compared with PCI patients. These factors may be relevant to the $50 \%$ of CABG strokes that occurred more than 30 days after operation.

Extensive post hoc subgroup analysis was undertaken to establish a role for the SYNTAX score, which is a risk stratification score based entirely on coronary anatomy and lesion characteristics. This score is not yet externally validated or published in detail, but it is known to contain risk adjustors that are not significant for CABG (in which coronary and lesion anatomy is largely irrelevant, as evidenced by registry results). The anatomic bias of the SYNTAX score may skew decisions toward the use of PCI despite other patient risk factors that support an advantage for CABG.
Opinion regarding the meaning and potential effect of the SYNTAX trial has been largely one-sided and aligned with the paradigm that as long as PCI has no demonstrable mortality disadvantage, it remains the treatment of choice. As described, however, absolutely no justification exists for even implying that PCI is equivalent to CABG for safety outcomes in this trial. In fact, the evidence supports the opposite conclusion. The trial was underpowered to demonstrate safety other than MACE (where CABG was shown to be significantly superior), and the mortality trend favors CABG, with $26 \%$ excess death at 1 year for PCI.

The paradigm of PCI equivalency also excludes the evidence from five observational trials of prospectively obtained nonrandomized clinical data ${ }^{5-9}$ indicating clinically and statistically significant excess mortality in "SYNTAX-like" patients when studied in sufficient numbers with sufficient duration. The hazard ratios for excess mortality with PCI range from 1.23 to 1.40 at 1 year, overlapping the SYNTAX result (1.26), and increase to 1.44 to 2.30 with 3- to 5-year follow-up. It is important to note that in SYNTAX, a mortality advantage trend for $C A B G$ was evident immediately and is likely to increase with further follow-up.

\section{APPROPRIATENESS CRITERIA}

Resolution of conflicting evidence and opinion by cardiology and surgeon experts working together has resulted in guidelines, but they are unavailable or only partially applicable for many clinical presentations. An effort to provide refined and extended guidance beyond that provided by evidence-based guidelines is presented in "Appropriateness Criteria for Coronary Revascularization" published simultaneously in the Journal of the American College of Cardiol$o g y^{2}$ and Circulation. ${ }^{3}$ This document is the result of a year-long effort to evaluate the available evidence and existing guidelines. An expert panel of 17 members, which included 4 cardiac surgeons and 4 interventional cardiologists, was constituted. Writing groups produced the vignettes. The 


\begin{tabular}{|c|c|c|c|c|c|c|}
\hline & \multicolumn{3}{|c|}{ CABG } & \multicolumn{3}{|c|}{$\mathrm{PCl}$} \\
\hline & \begin{tabular}{|c|}
$\begin{array}{c}\text { To diabetes } \\
\text { and normal } \\
\text { LVEF }\end{array}$ \\
\end{tabular} & Diabetes & $\begin{array}{c}\text { Depressed } \\
\text { LVEF }\end{array}$ & \begin{tabular}{|c|}
$\begin{array}{c}\text { Ho diabetes } \\
\text { and normal } \\
\text { LVEF }\end{array}$ \\
\end{tabular} & Diabetes & $\begin{array}{c}\text { Depressed } \\
\text { LVEF }\end{array}$ \\
\hline $\begin{array}{l}\text { Two vessel coronary artery disease } \\
\text { with proximal LAD stenosis }\end{array}$ & A & A & A & A & A & A \\
\hline Three vessel coronary artery disease & A & A & A & u & u & u \\
\hline Isolated left main stenosis & A & A & A & I & $\mathbf{I}$ & I \\
\hline $\begin{array}{l}\text { Left main stenosis and additional } \\
\text { coronary artery disease }\end{array}$ & A & A & A & I & I & I \\
\hline
\end{tabular}

FIGURE 2. Appropriateness criteria result for percutaneous coronary intervention (PCI) or coronary artery bypass grafting (CABG) in patients with coronary artery disease extent considered sufficient for appropriateness of revascularization, stratified by extent of disease and association of diabetes or impaired left ventricular function. A, Appropriate; $U$, uncertain; $I$, inappropriate; $L A D$, left anterior descending artery; $L V E F$, left ventricular ejection fraction. This figure is reproduced, with permission, from “Appropriateness Criteria for Coronary Revascularization" by Patel MR, et al. J Am Coll Cardiol. 2009;53:545. Copyright 2009 American College of Cardiology Foundation.

vignettes were scored for the appropriateness of revascularization (PCI or CABG) on a scale of 1 (inappropriate) to 9 (appropriate). PCI and CABG were scored independently for vignettes describing severe $\mathrm{CAD}$ with and without ventricular dysfunction or diabetes. The scores were used to rate each vignette as appropriate ( 7 to 9), uncertain (4 to 6), or inappropriate (1 to 3 ) based on the median score obtained. The entire expert panel and writing group then met, using a modified Delphi method. All results were discussed, and a second and final round of voting was conducted to produce the recommendations.

In general, PCI was rated as appropriate for acute myocardial injury and most scenarios where there was significant CAD associated with demonstrable ischemia and symptoms despite adequate medical therapy. The degree of appropriateness generally increased as the extent of myocardium at risk increased until the 3-vessel disease threshold of CAD extent was reached. For these patients, PCI was rated as uncertain regardless of the presence of diabetes or left ventricular dysfunction. PCI for left main CAD was scored as inappropriate in all described settings. CABG was rated as appropriate for 3 -vessel and for left main CAD in all settings (Fig 2).

\section{EVIDENCE VS THE BELIEF SYSTEM}

In summary, there are consistent and abundant data to establish that CABG is the standard of care for 3-vessel disease or left main disease, or both. Yet, it is the perception of PCI effectiveness rather than published evidence of comparative effectiveness (equivalency or noninferiority) of PCI to CABG that are in play today. These perceptions have induced the principal decision makers - cardiologists and patients - to believe PCI is the standard of care for most patients, even those with severe CAD. These perceptions range from the sublime:

... the trial design actually stacked the cards against PCI intervention by combining both the hard end points of death, MI, and stroke, and the soft end point, the need for revascularization, into a combined primary end point. If the trial design had separated those end points, the primary end point would have been equivalent ... On the one hand, CABG led to more complete revascularization with a higher stroke rate and, I'd also point out, a higher noncardiac mortality rate. On the other hand, we know PCI leads to less complete revascularization, perhaps a numerical difference in cardiac mortality, but a smaller stroke rate, and certainly easier on the individual, in terms of procedure recovery time and other factors". ${ }^{10}$

to the ridiculous:

$\ldots$ it would take a very unlikely $\geq 50 \%$ increase in mortality in the stent arm of the upcoming randomized trials before $\mathrm{CABG}$ becomes close to an unequivocal treatment choice. ... Most of my patients would rather have 2,3 , or even 5 stent procedures to avoid 1 bypass surgery. ${ }^{11}$

Unfortunately, the end result has been to reconcile all evidence to favor PCI and conserve a faulty belief system. The resulting changes in treatment selection during the past 8 years [unpublished data from the Duke Cardiovascular Disease Databank] clearly illustrate this evolution (Fig 3).

The editorial associated with the SYNTAX trial publication $^{12}$ is generally balanced and calls for separating CAD diagnosis and treatment to allow for multidisciplinary decision making regarding treatment selection. Recommendations must be guided by a patient-centric process that results in reasoned agreement between colleagues rather than procedural advocacy. It is incumbent upon thoracic surgeons to "own" this disease through knowledge and experience and not own the patient or the procedure. This paradigm includes a responsibility to ensure that $\mathrm{CABG}$ patients receive optimal medical management for the rest of their lives.

Although the evidence supports the appropriateness of CABG for virtually all scenarios of significant CAD, it is no more the treatment of choice for all patients than is PCI. CABG should be the baseline choice for 3-vessel 


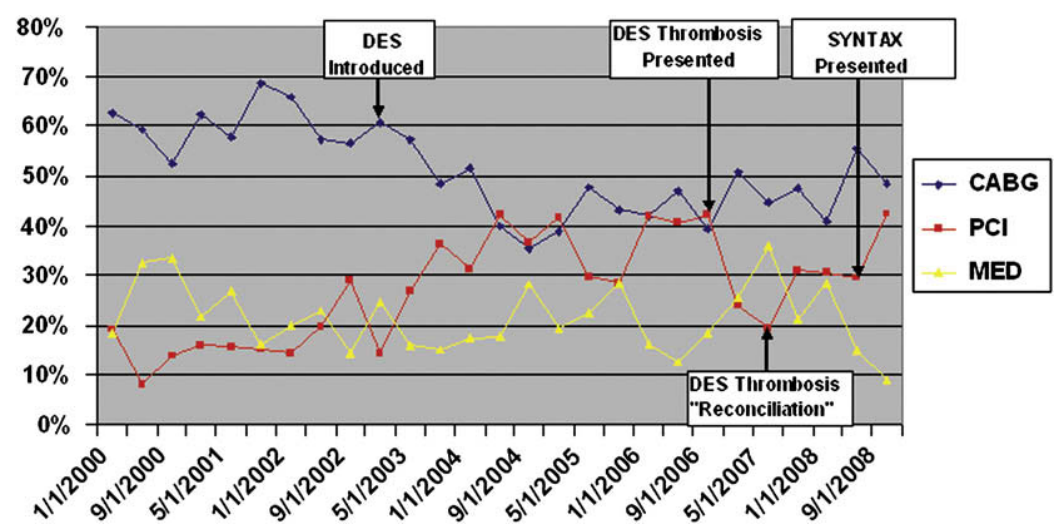

FIGURE 3. Changing patterns of initial treatment selection among medical therapy (MED, yellow line), percutaneous coronary intervention (PCI, red line), and coronary artery bypass grafting $(C A B G$, blue line ) from 2000 to 2008 at Duke University in 10,149 patients. Decline in CABG selection is seen after drugeluting stent (DES) introduction, with a corresponding increase in PCI. The presentation of DES acute thrombosis as a concern resulted in a decline in PCI but an increase in medical therapy selection rather than CABG. Reconciliation occurred through observational analysis and expert opinion that indefinite dual antiplatelet therapy and decreasing DES use may limit acute stent thrombosis. With the presentation of the Synergy between PCI with Taxus and Cardiac Surgery (SYNTAX) trial, PCI recommendations have returned to 2004 levels.

disease and for almost all patients with left main disease, but PCI has a definite role as the best choice for some patients. This is consistent with the "Appropriateness Criteria for Coronary Revascularization" recommendations.

PCI should be considered in 3-vessel or left main disease when the CABG benefit is likely to be offset by advanced comorbidity, as determined by the cardiologist and surgeon. These more complex patients should be further defined by coronary anatomic features. Treatment recommendations, which may include off-pump or hybrid procedures, should be based on the relative risk of PCI failure compared with CABG failure.

CABG should be the predominant recommendation for these patients unless there is a substantially increased risk of CABG, such as age, prior stroke, reoperative status, ongoing myocardial injury, that does not simultaneously add benefit (eg, diabetes, impaired ventricular function). As documented by the SYNTAX trial, CABG is largely independent of coronary vascular anatomy (198 exclusions referred to PCI, for comorbidity), whereas anatomy commonly increases the procedural risks of PCI (1077 exclusions referred for CABG).

The goal is to achieve the greatest benefit for the patient with the least risk, and this can only be achieved by communication and cooperation between cardiac surgeons and cardiologists. It is imperative that this be framed using only one belief system constructed by evidence that is carefully and fairly judged. It is imperative that all providers participate, so that patients with 3-vessel disease or left main disease are treated according to the available evidence, because their lives depend on it.

\section{References}

1. Serruys PW, Morice MC, Kappetein AP, et al., SYNTAX Investigators. Percutaneous coronary intervention versus coronary-artery bypass grafting for severe coronary artery disease. $N$ Engl J Med. 2009;360:961-72.
2. Patel MR, Dehmer GJ, Hirshfeld JW, et al. ACCF/SCAI/STS/AATS/AHA/ ASNC 2009 appropriateness criteria for coronary revascularization: a report by the American College of Cardiology Foundation Appropriateness Criteria Task Force, Society for Cardiovascular Angiography and Interventions, Society of Thoracic Surgeons, American Association for Thoracic Surgery, American Heart Association, and the American Society of Nuclear Cardiology endorsed by the American Society of Echocardiography, the Heart Failure Society of America, and the Society of Cardiovascular Computed Tomography. J Am Coll Cardiol. 2009;53:530-53.

3. Patel MR, Dehmer GJ, Hirshfeld JW, et al. ACCF/SCAI/STS/AATS/AHA/ ASNC 2009 Appropriateness criteria for coronary revascularization: a report of the American College of Cardiology Foundation Appropriateness Criteria Task Force, Society for Cardiovascular Angiography and Interventions, Society of Thoracic Surgeons, American Association for Thoracic Surgery, American Heart Association, and the American Society of Nuclear Cardiology: endorsed by the American Society of Echocardiography, the Heart Failure Society of America, and the Society of Cardiovascular Computed Tomography. Circulation. 2009; 119:1330-52.

4. Morrison DA, Sethi G, Sacks J, et al. Percutaneous coronary intervention versus coronary artery bypass graft surgery for patients with medically refractory myocardial ischemia and risk factors for adverse outcomes with bypass: a multicenter, randomized trial: Investigators of the Department of Veterans Affairs Cooperative Study \#385, the Angina With Extremely Serious Operative Mortality Evaluation (AWESOME). J Am Coll Cardiol. 2001;38:143-9.

5. Brener SJ, Lytle BW, Casserly IP, Schneider JP, Topol EJ, Lauer MS. Propensity analysis of long-term survival after surgical or percutaneous revascularization in patients with multivessel coronary artery disease and high-risk features. Circulation. 2004;109:2290-5.

6. Malenka DJ, Leavitt BJ, Hearne MJ, et al. Comparing long-term survival of patients with multivessel coronary disease after CABG or PCI: analysis of BARI-like patients in northern New England. Circulation. 2005; 112(9 suppl):I371-6.

7. Hannan EL, Racz MJ, Walford G, et al. Long-term outcomes of coronary-artery bypass grafting versus stent implantation. N Engl J Med. 2005;352:2174-83.

8. Smith PK, Califf RM, Tuttle RH, et al. Selection of surgical or percutaneous coronary intervention provides differential longevity benefit. Ann Thorac Surg. 2006;82:1420-8; discussion 1428-9.

9. Hannan EL, Wu C, Walford G, et al. Drug-eluting stents vs. coronary-artery bypass grafting in multivessel coronary disease. N Engl J Med. 2008;358:331-41.

10. Lee TH, Hillis LD, Nabel EG. CABG vs. stenting-clinical implications of the SYNTAX trial. $N$ Engl J Med. 2009;360:e10.

11. Teirstein PS. Percutaneous revascularization is the preferred strategy for patients with significant left main coronary stenosis. Circulation. 2009; 119:1021-33.

12. Lange RA, Hillis LD. Coronary revascularization in context. $N$ Engl $J$ Med 2009;360:10. 